\title{
Non-Local Means Variants for Denoising of Diffusion-Weighted and Diffusion Tensor MRI
}

\author{
Nicolas Wiest-Daesslé, Sylvain Prima, Pierrick Coupé, Sean Patrick Morrissey, \\ and Christian Barillot \\ Unit/Project VisAGeS U746, INSERM - INRIA - CNRS - Univ-Rennes 1, \\ IRISA campus Beaulieu 35042 Rennes Cedex, France \\ \{nwiestda, sprima, pcoupe, spmorris, cbarillo\}@irisa.fr \\ http://www.irisa.fr/visages
}

\begin{abstract}
Diffusion tensor imaging (DT-MRI) is very sensitive to corrupting noise due to the non linear relationship between the diffusionweighted image intensities (DW-MRI) and the resulting diffusion tensor. Denoising is a crucial step to increase the quality of the estimated tensor field. This enhanced quality allows for a better quantification and a better image interpretation. The methods proposed in this paper are based on the Non-Local (NL) means algorithm. This approach uses the natural redundancy of information in images to remove the noise. We introduce three variations of the NL-means algorithms adapted to DW-MRI and to DT-MRI. Experiments were carried out on a set of 12 diffusion-weighted images (DW-MRI) of the same subject. The results show that the intensity based NL-means approaches give better results in the context of DT-MRI than other classical denoising methods, such as Gaussian Smoothing, Anisotropic Diffusion and Total Variation.
\end{abstract}

\section{Introduction}

Image processing procedures needed for fully automated and quantitative analysis (registration, segmentation, visualisation) require images with the best signalto-noise ratio and the least artifacts in order to improve their performances. Most of the time, the hardware introduces artifacts during the acquisition (noise, intensity non-uniformities, geometrical deformations). Therefore, one critical issue is to remove the noise while keeping relevant image information. This is particularly true for diffusion-weighted MRI (DW-MRI) especially when they are acquired with high diffusion ( $b$-value) coefficient. This paper focuses on denoising using variants of the non-local means (NL-means) method modified to deal with DT-MRI (NLMt) and DW-MRI, either gradient-by-gradient (NLM) or as a multi-spectral (NLMv) image. The NL-means variants are compared with the simple Gaussian Filter (GF), the Anisotropic Diffusion (AD) [13] and the Total Variation (TV) [15. In particular the AD filter is frequently used for diffusion image denoising [4,9] or tensor field regularisation [16]. 


\section{Methods}

\subsection{The Non-Local Means Algorithm}

First introduced by Buades et al. in 3 , the NL-means algorithm is based on the natural redundancy of information in images to remove noise. In the theoretical formulation of the NL-means algorithm, the restored intensity of the voxel $x_{i}, N L(v)\left(x_{i}\right)$, is a weighted average of all voxels intensities in the image $I$. Let us denote:

$$
N L(v)\left(x_{i}\right)=\sum_{x_{j} \in I} w\left(x_{i}, x_{j}\right) v\left(x_{j}\right),
$$

where $v$ is the intensity function and thus $v\left(x_{j}\right)$ is the intensity of voxel $x_{j}$ and $w\left(x_{i}, x_{j}\right)$ the weight assigned to $v\left(x_{j}\right)$ in the restoration of $v\left(x_{i}\right)$. More precisely, the weight quantifies the similarity of voxels $x_{i}$ and $x_{j}$ under the assumptions that $w\left(x_{i}, x_{j}\right) \in[0,1]$ and $\sum_{x_{j} \in I} w\left(x_{i}, x_{j}\right)=1$.

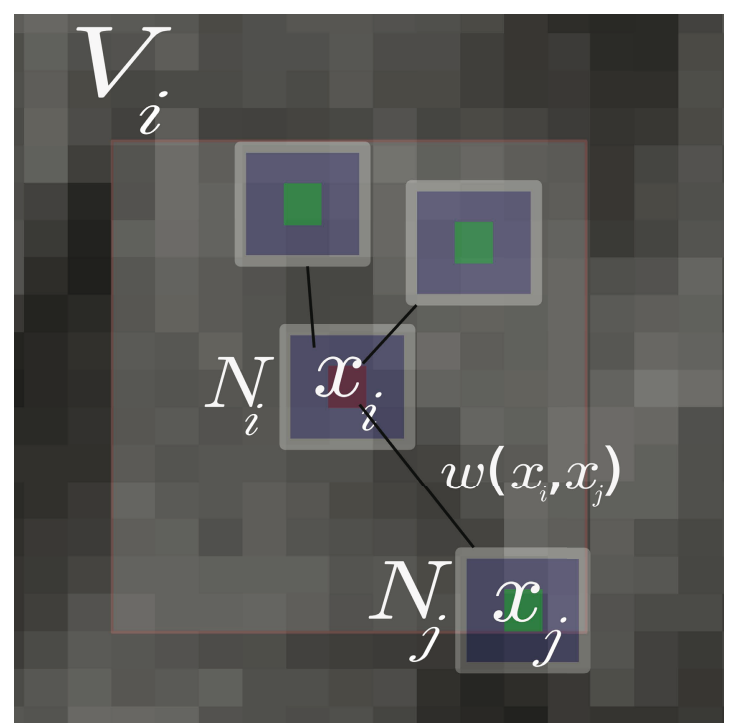

Fig. 1. Two-dimensional illustration of the NL-means principle. The restored value of voxel $x_{i}$ is a weighted average of all intensities of voxels $x_{j}$ in the search volume $V_{i}$. The weight $w\left(x_{i}, x_{j}\right)$ is based on the similarity of the intensities in cubic neighborhoods $N_{i}$ and $N_{j}$ around $x_{i}$ and $x_{j}$.

In practice, voxels similar to $i$ are only searched over a neighborhood $V_{i}$, so Eq. 1 is: $N L(v)\left(x_{i}\right)=\sum_{x_{j} \in V_{i}} w\left(x_{i}, x_{j}\right) v\left(x_{j}\right)$. For each voxel $x_{j}$ in $V_{i}$, the weight $w\left(x_{i}, x_{j}\right)$ is related to the distance $d\left(v\left(N_{i}\right), v\left(N_{j}\right)\right), N_{i}$ and $N_{j}$ being neighborhoods around $x_{i}$ and $x_{j}$, following:

$$
w\left(x_{i}, x_{j}\right)=\frac{1}{Z(i)} e^{-\frac{d\left(v\left(N_{i}\right), v\left(N_{j}\right)\right)}{(h \hat{\sigma})^{2}}}
$$


where $Z(i)$ is a normalization constant with $Z(i)=\sum_{j} w\left(x_{i}, x_{j}\right), \hat{\sigma}$ is the estimation of the standard deviation of the noise using the pseudo-residuals method [8] and $h$ acts as a filtering parameter (for more details see [6] and Fig. 11). The distance $d$ is expressed in general terms as: $d\left(v\left(N_{i}\right), v\left(N_{j}\right)\right)=$ $\sqrt{\frac{1}{N} \sum_{k}^{N} \Delta\left(v\left(y_{k}\right), v\left(z_{k}\right)\right)}$ where $N=\operatorname{card} N_{i}=\operatorname{card} N_{j}$ and $y_{k}$ and $z_{k}$ are the $k$-th voxels in the neighborhoods $N_{i}$ and $N_{j}$. For a grey-level image, $\Delta$ is $\Delta\left(v\left(y_{k}\right), v\left(z_{k}\right)\right)=\left\|v\left(y_{k}\right)-v\left(z_{k}\right)\right\|^{2}$.

\subsection{DW- and DT-MRI Adaptations}

This section introduces the NL-means as a method to remove noise from either the whole DW-MR dataset (with $n$ directions, $n \geq 6$, plus the $B_{0}$ image) or the resulting DT-MR image. Three variants are proposed here, two acting on the DW-MRI and one on the DT-MRI:

1. NLM: each DW-MRI is denoised individually as described in Section 2.1 and the DT-MRI is estimated from these denoised DW-MRI,

2. NLMv: the whole set of DW-MRI is considered as a multi-spectral image, each voxel being a $(n+1)$-dimensional vector. The $\Delta$ is defined as:

$$
\Delta\left(v\left(y_{k}\right), v\left(z_{k}\right)\right)=\sum_{i=1}^{n+1}\left\|v^{i}\left(y_{k}\right)-v^{i}\left(z_{k}\right)\right\|^{2},
$$

$v^{i}$ (.) being the $i$-th component of the vector $v($.$) .$

3. NLMt: the DT-MRI is computed from the raw DW-MRI and then denoised. The weighted average of the MRI intensities (grey levels) Eq. 1 is replaced by a Log-Euclidean weighted average [1,12]: of the image diffusion tensors. $\Delta$ is defined as:

$$
\Delta\left(v\left(y_{k}\right), v\left(z_{k}\right)\right)=\left\|\log \left(v\left(y_{k}\right)^{-\frac{1}{2}} v\left(z_{k}\right) v\left(y_{k}\right)^{-\frac{1}{2}}\right)\right\|^{2},
$$

$v\left(y_{k}\right)$ and $v\left(z_{k}\right)$ being the tensors at voxels $y_{k}$ and $z_{k}$.

The Log-Euclidean framework could have been replaced by other methods [7, 12, 17.

\subsection{Implementation Details}

The NLM method uses a cubic neighborhood $\left(\operatorname{card} N_{i}=27\right)$. For NLMv and NLMt, considering that both vectors and tensors convey enough information for denoising, card $N_{i}$ is set to 1 . In these cases, having larger neighborhoods $N_{i}$ makes it difficult to find similar blocks in the search area $V_{i}$ and thus limits the denoising capacities of the algorithms. The search area $V_{i}$ is chosen to be identical for all the NL-means variants ( $\operatorname{card} V_{i}=11^{3}$ voxels). 


\subsection{Comparison Measure}

A comparison measure is needed to validate the denoising methods with respect to a ground truth. We define the distance between two DT-MRI as:

$$
R M S=\sqrt{\frac{1}{\operatorname{card} \Omega} \sum_{\operatorname{card} \Omega} d\left(\widehat{I}, I_{d}\right)^{2}},
$$

with $\Omega$ the masked diffusion tensor image grid, $\widehat{I}$ the reference DT-MRI, $I_{d}$ the denoised DT-MRI, and $d$ a distance over tensors. The Log-Euclidean distance is selected as it is specifically designed for tensors as described in Sec. 2.2. The comparison is restricted to cerebral tissues, where the estimation of a diffusion tensor is relevant.

\section{$3 \quad$ Validation and Results}

\subsection{Dataset}

In order to evaluate the performances of the different algorithms on DT-MRI multiple tests are performed on a reference data set. The reference data set is constructed by averaging multiple acquisitions of the same subject. The acquisition protocol is a single-shot spin echo EPI sequence on a Siemens 1.5T scanner, with diffusion encoding (10 directions, $b=1000 \mathrm{~s} / \mathrm{mm}^{2}$, voxels $=1.875 \times 1.875 \times 5$ $\mathrm{mm}^{3}, 24$ slices, $24 \mathrm{~cm}$ FOV). The acquisition is repeated 12 times with identical slice locations and each acquisition has a run time of 8 minutes. Each diffusionweighted acquisition is corrected for distortions [18.

Numerous methods exist for the estimation of the tensor [10,16]. We simply choose to estimate the tensor by classical linear regression.

\subsection{Leave-One-Out Comparison}

To assess the validity of the proposed denoising methods, a leave-one-out approach is devised. For each DW acquisition $I_{\text {noisy }}^{i}$ the 11 other DW-MRI are averaged gradient-by-gradient, giving $I_{\text {average ( }}^{i} f$ Fig. 2. left). A DT-MRI is estimated from $I_{\text {average }}^{i}$ and serves as a comparison basis. The selected image, $I_{\text {noisy }}^{i}$ (or its corresponding DT-MRI), is then denoised with the 6 denoising techniques, and the resulting denoised DT-MRI is estimated. The error between this denoised image and the ground truth data built from $I_{\text {average }}^{i}$ is computed using the measure defined in Section 2.4. The process is then iterated, yielding 12 RMS errors, which are finally averaged to give a global RMS error. These error measures are displayed on Fig. 2 (right) for the 6 denoising methods. This leave-one-out method helps avoiding the introduction of bias.

The denoising using the NLMt techniques yields very poor results, probably due to the poor redundancy of tensor information in the image. Computing the 

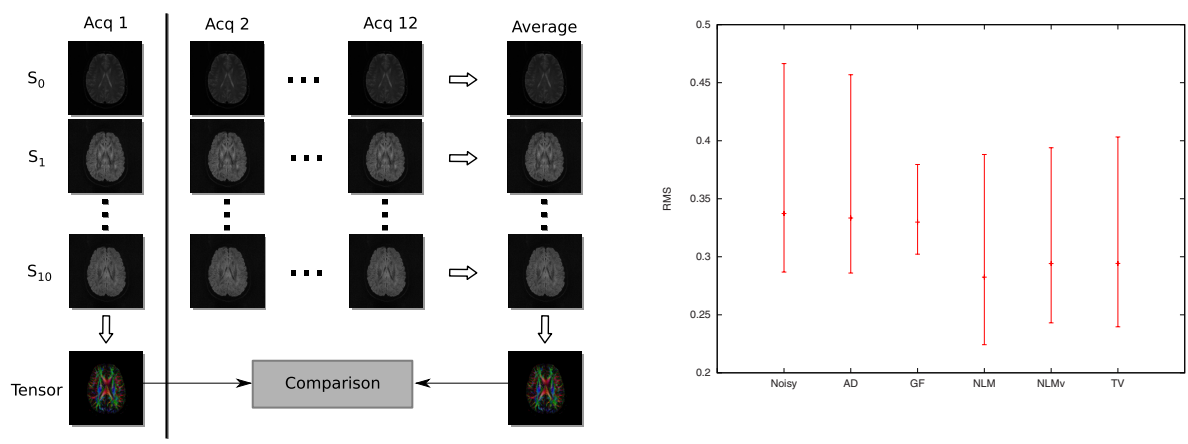

Fig. 2. Left: Scheme of the first step of the leave-one-out validation. An acquisition is selected $\left(I_{\text {noisy }}^{1}\right)$, and the others are averaged, giving $I_{\text {average. The DW-MRI }}^{1}$ (or DT-MRI) corresponding to $I_{\text {noisy }}^{1}$ are denoised with each of the 6 algorithm; the associated DT-MRI is computed and quantitatively compared with the DT-MRI com-

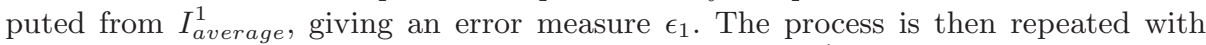
$i=2, \ldots, 12$ and the global error measure is computed as $\frac{1}{12} \sum_{i} \epsilon_{i}$. Right: Error plot of the RMS for each method. The bar length indicates the min and max error over the 12 experiments; the middle mark indicates the mean value. The acronyms are as follows: GF: Gaussian Filter, AD: Anisotropic Diffusion, TV: Total Variation, NLMv: NL-means vector, NLM: NL-means gradient-by-gradient, NLMt: NL-means tensor. The NLMt method is not plotted due to poor results: average RMS is 1.2.

weights for each voxel shows that on average only 8 significantly similar tensors are found, whereas for grey level or vector images the number of significantly similar blocks is generally higher than 100 .

\subsection{Comparison with Different Noise Levels}

In this section, the average of the 12 images $I_{a v g}$ is used as a reference. A new image $I_{n}$ is built by adding Rician noise with different levels. In Collins et al. [5], the noise percentage $p$ is related to the standard deviation of the Gaussian noise $\sigma$ and the mean value $\nu$ of the brightest tissue following $p=100 \sigma / \nu$. The same idea is used here with Rician noise. The mean intensity of the CSF in the nondiffusion-weighted image $\left(S_{0}\right)$ is used for the $\nu$ value. The RMS error is computed between each denoised DT-MRI and the ground truth DT-MRI computed from Iaverage.

Results are shown in Fig. 3. At low levels of noise (below 4\%), TV and AD perform better than the NL-means filters. That could be partially explained by the fact that the estimation of the noise by pseudo-residuals used in the NL-means variants is known to be overestimated for these low levels of noise. At higher levels (in the range 5-10\%), usually met in real DW-MRI, the NLmeans filters outperform all the other filters, NLM being constantly better than NLMv. 


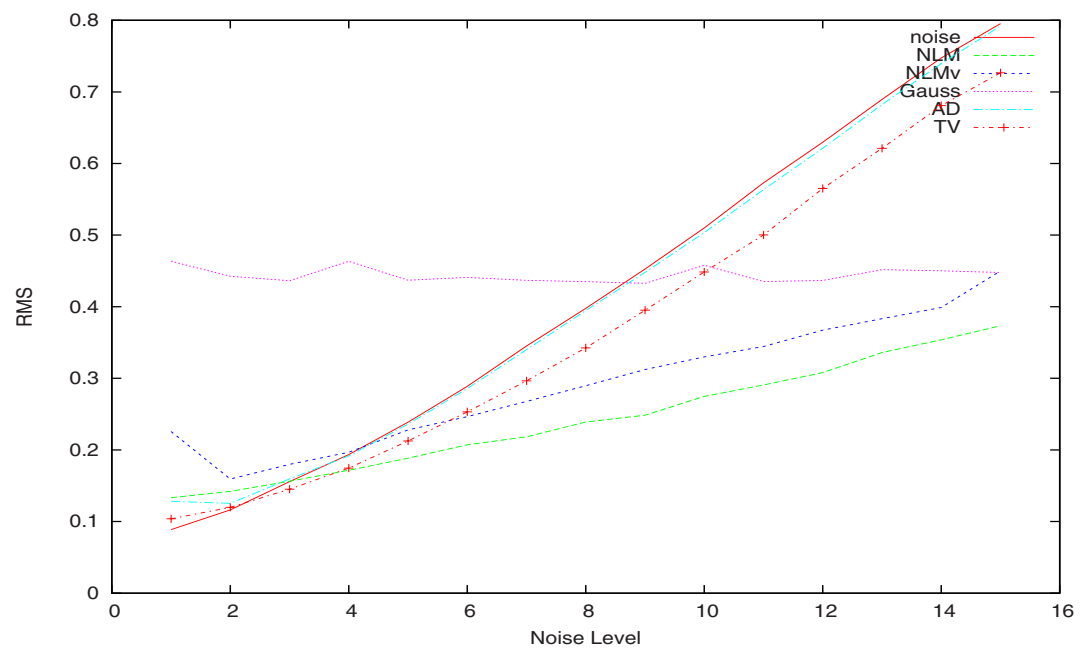

Fig. 3. Plot of different noise levels and RMS. Noise is added to the reference image. The image is then denoised and compared to the original.

\subsection{Choice of the Filters Parameters}

Each proposed method needs specific parameters for denoising. For a fair comparisons of all the methods, those parameters are selected with an optimisation procedure so that each method gives its best result for a given experiment. In practice, according to the 12 different RMS errors computed in the leave-one-out experiment, the noise level is between 6 and 7 percents. All the parameters are optimised for this level of noise added to the $I_{\text {average }}$ DW-MRI.

This optimisation is performed with the Nelder-Mead's downhill simplex algorithm [14]. The cost function for this optimiser is the measure described in Sec. 2.4. Initial guesses for the parameters are empirically chosen after a few manual tests, and are used to initialise the downhill simplex. The unknown parameters are: number of iterations and regularisation strength ( $T V$ and $\mathrm{AD}$ ), kernel size (GF), and filtering parameter $h$ (NL-means variants).

\subsection{Visual Assessment}

In Figure 4 we display axial slices at the level of the ventricles for the ground truth data (DT-MRI computed from $I_{\text {average }}$ ), the raw DT-MRI computed from one of the acquisitions $I_{\text {noisy }}^{i}$, and the 6 denoised images. The color encodes the principal direction of diffusion (colinear to the eigenvector of the tensor with maximal eigenvalue), weighted by the fractional anisotropy [11. The reference image has smooth color transitions but also sharp edges. The GF filtered image efficiently removes the noise but suppresses the edges and lowers the anisotropy of tensors. The standard NL-means seems to be the best filter, followed by TV, NLMv, AD and NLMt, which confirms the quantitative values in Fig. 3 , 


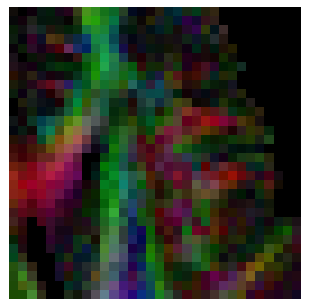

Noisy

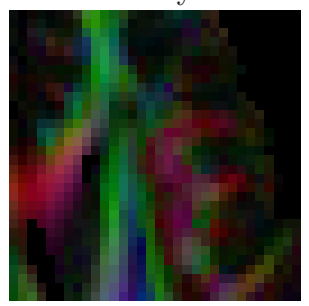

Reference

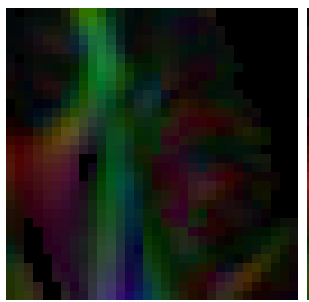

GF

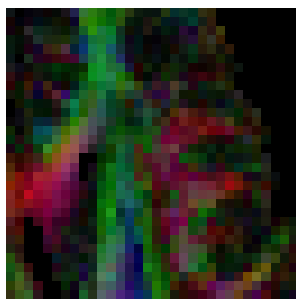

$\mathrm{AD}$

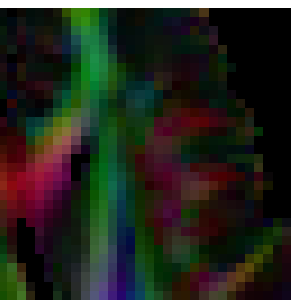

NLM

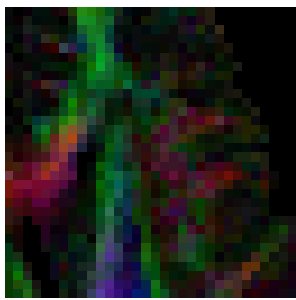

NLMt

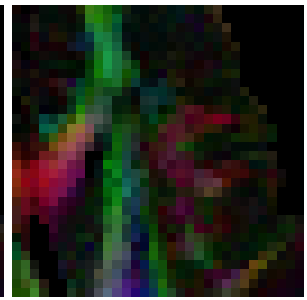

NLMv

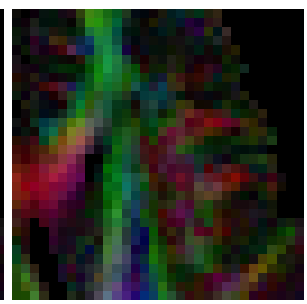

TV

Fig. 4. Visual Comparison of the different algorithms. The color encodes the principal direction of diffusion (colinear to the eigenvector of the tensor with maximal eigenvalue), weighted by the fractional anisotropy [1].

\section{Conclusion and Further Works}

This paper presents new variants of the Non-Local (NL) means algorithm, applied to diffusion-weighted and diffusion tensor images. The validations performed on a reference dataset underline how the NL-means denoising outperforms well-established other methods, such as Anisotropic Diffusion 13. and Total Variation [15. The results are obtained from the denoising of either the diffusion images or the diffusion tensor. Our comparison does not take into account the Rician nature of the noise, and comparison with more specific denoising methods 2 will be performed in the future. The direct denoising of the DT-MRI with our proposed NL-means variant does not achieve good performances. This relates to the fact that the number of similar tensors inside the search region is quite low $(\approx 8)$. The lower quality of the direct denoising of DT-MRI compared to denoising on DW-MRI is in line with the literature [2].

The effect of such denoising techniques needs to be investigated in pathological cases. For instance Multiple Sclerosis clearly shows changes in diffusion coefficients (such as fractional anisotropy and mean diffusivity). The effect of denoising must be studied in lesion areas to make sure these are well preserved in terms of their diffusion characteristics. Moreover, the impact of this NL-means denoising variants on the performances of post-processing algorithms, such as segmentation and fiber tracking has to be further investigated. 


\section{References}

1. Arsigny, V., Fillard, P., Pennec, X., Ayache, N.: Fast and simple calculus on tensors in the Log-Euclidean framework. In: Duncan, J.S., Gerig, G. (eds.) MICCAI 2005. LNCS, vol. 3749, pp. 115-122. Springer, Heidelberg (2005)

2. Basu, S., Fletcher, T., Whitaker, R.: Rician noise removal in diffusion tensor mri. In: Larsen, R., Nielsen, M., Sporring, J. (eds.) MICCAI 2006. LNCS, vol. 4190, pp. 117-125. Springer, Heidelberg (2006)

3. Buades, A., Coll, B., Morel, J.M.: A review of image denoising algorithms, with a new one. Multiscale Modeling \& Simulation 4(2), 490-530 (2005)

4. Chen, B., Hsu, E.: Pde denoising of MR diffusion tensor imaging data. In: ISBI 2004, pp. 1040-1042 (2004)

5. Collins, D.L., Zijdenbos, A.P., Kollokian, V., Sled, J.G., Kabani, N.J., Holmes, C.J., Evans, A.C.: Design and construction of a realistic digital brain phantom. IEEE Trans. Med. Imaging 17(3), 463-468 (1998)

6. Coupé, P., Yger, P., Barillot, C.: Fast Non Local Means Denoising for 3D MR Images. In: Larsen, R., Nielsen, M., Sporring, J. (eds.) MICCAI 2006. LNCS, vol. 4191, pp. 33-40. Springer, Heidelberg (2006)

7. Fletcher, P.T., Joshi, S.C.: Principal geodesic analysis on symmetric spaces: statistics of diffusion tensors. In: Sonka, M., Kakadiaris, I.A., Kybic, J. (eds.) CVAMIA and MMBIA 2004. LNCS, vol. 3117, pp. 87-98. Springer, Heidelberg (2004)

8. Gasser, T., Sroka, L., Steinmetz, C.: Residual variance and residual pattern in non linear regression. Biometrika 73(3), 625-633 (1986)

9. Lee, J.E., Chung, M.K., Alexander, A.L.: Evaluation of anisotropic filters for diffusion tensor imaging. In: on Biomedical Imaging: Macro to Nano 3rd IEEE International Symposium, pp. 77-78. IEEE Computer Society Press, Los Alamitos (2006)

10. Mangin, J.-F., Poupon, C., Clark, C., Le Bihan, D., Bloch, I.: Distortion correction and robust tensor estimation for MR diffusion imaging. Med. Image Anal. 6(3), 191-198 (2002)

11. Pajevic, S., Pierpaoli, C.: Color schemes to represent the orientation of anisotropic tissues from diffusion tensor data: application to white matter fiber tract mapping in the human brain. Magn. Reson. Med. 42(3), 526-540 (1999)

12. Pennec, X., Fillard, P., Ayache, N.: A Riemannian framework for tensor computing. International Journal of Computer Vision 66(1), 41-66 (2006)

13. Perona, P., Malik, J.: Scale-space and edge detection using anisotropic diffusion. IEEE Trans. Pattern Anal. Mach. Intell. 12(7), 629-639 (1990)

14. Press, W.H., Flannery, B.P., Teukolsky, S.A., Vetterling, W.T.: Numerical Recipes: The Art of Scientific Computing, 2nd edn. Cambridge University Press, Cambridge (UK), New York (1992)

15. Rudin, L.I., Osher, S., Fatemi, E.: Nonlinear total variation based noise removal algorithms, pp. 259-268 (1992)

16. Tschumperlé, D., Deriche, R.: Variational frameworks for DT-MRI estimation, regularization and visualization. In: ICCV 2003, pp. 116-121 (October 2003)

17. Wang, Z., Vemuri, B.C.: DTI segmentation using an information theoretic tensor dissimilarity measure. IEEE Trans. Med. Imaging 24(10), 1267-1277 (2005)

18. Wiest-Daesslé, N., Prima, S., Morrissey, S.P., Barillot, C.: Validation of a new optimisation algorithm for registration tasks in medical imaging. In: ISBI 2007 (April 2007) 